\title{
Internet der Energie
}

\author{
IKT als Schlüsseltechnologie \\ für das Energiesystem der Zukunft
}

\section{Die Autoren}

\author{
Prof. Dr. Dr. h.c. \\ Hans-Jürgen Appelrath \\ Fakultät II \\ Department für Informatik \\ Universität Oldenburg \\ Escherweg 2 \\ 26121 Oldenburg \\ Deutschland \\ appelrath@office.de
}

Prof. Dr. Orestis Terzidis ( $\varangle)$

Fakultät für

Wirtschaftswissenschaften Institut für Entrepreneurship, Technologie-Management und Innovation (EnTechnon) Karlsruhe Institute of Technology (KIT)

Engesser Str. 9

76131 Karlsruhe

Deutschland

orestis.terzidis@kit.edu

Prof. Dr. Christof Weinhardt

Fakultät für

Wirtschaftswissenschaften

Institut für Informationswirtschaft

und -management

Karlsruhe Institute of Technology

(KIT)

Englerstraße 14

76131 Karlsruhe

Deutschland

christof.weinhardt@kit.edu

Online publiziert: 2012-01-14

This article is also available in English via http://www.springerlink.com and http://www.bise-journal.org: Appelrath $\mathrm{H}-\mathrm{J}$, Terzidis $\mathrm{O}$, Weinhardt $\mathrm{C}$ (2011). Internet of Energy. ICT as a Key Technology for the Energy System of the Future. Bus Inf Syst Eng. doi: 10.1007/s12599-011-0197-x.

(C) Gabler Verlag 2011

\section{DOI 10.1007/s11576-011-0304-0}

Seit dem Erscheinen des von Appelrath und Chamoni herausgegebenen Schwerpunktthemas „IT in der Energiewirtschaft“ (WI 49) der Zeitschrift WIRTSCHAFTSINFORMATIK im Herbst 2007 hat eine dynamische Entwicklung in dem Feld stattgefunden.

Getrieben durch die fortschreitenden Folgen der Liberalisierung und den forcierten Ausbau der erneuerbaren Energien entstand eine nachhaltige Marktdynamik, die die Transformation der Branche weiter vorangebracht hat.

Parallel hierzu fand mit dem seit 2008 laufenden Forschungsprogramm E-Energy der Bundesregierung und den in diesem Rahmen angestrengten Projekten der sechs Modellregionen eine systematische explorative und empirische Forschung statt, bei der verschiedene Konzepte entwickelt und in ausgedehnten Feldversuchen erprobt wurden. Auch die Forschung im Bereich der Infrastrukturen und Prozesse für die Elektromobilität spielte eine wichtige Rolle in der Forschungslandschaft der letzten Jahre.

Die größte Dynamik kommt aber wohl aus der politischen Entwicklung der letzten Monate. Die Bundesregierung legte im Herbst 2010 ihr Energiekonzept vor, bei dem der Ausbau erneuerbarer Energien zu einer zentralen Rolle des Energiesystems dargestellt , aber auch die Nutzung der Kernenergie als „Brückentechnologie“ für die kommenden beiden Jahrzehnte festgeschrieben wurde. Mit der Reaktorkatastrophe in Fukushima wurde die Situation neu bewertet und in der Folge mit der Novellierung des Atomgesetzes (AtG) der beschleunigte Ausstieg aus der Kernenergie beschlossen. Der Bundestag verabschiedete das Gesetz am 30. Juni 2011 mit großer Mehrheit und entschied so eine Diskussion, die in Deutschland über drei Jahrzehnte zum festen Bestandteil der politischen Debatte geworden war. Mit der flankierenden Novellierung des Energiewirtschaftsgesetzes (EnWG) wurden am 28. Juli 2011 die Weichen für die Neugestaltung des Energiesystems gestellt.

Diese Transformation des Energiesystems umfasst eine ganze Reihe von Faktoren und Parametern. Die Erzeugung, der Transport, die Speicherung und der Verbrauch von Energie lassen sich auf der Seite der Primärtechnologien unterscheiden. Im System liegen dabei ganz verschiedene Größenordnungen vor - von Gigawatt bei Großkraftwerken, großen Übertragungsnetzen und Großverbrauchern (wie z. B. Aluminiumwerken) über Megawatt von Kraftwerken für Städte und Industrieanlagen bis hinunter zur Kilowatt auf der Ebene der Verteilnetze, häuslichen Kraft-Wärme-Kopplungs- und Photovoltaikanlagen besteht ein breites Spektrum von Geräten und Infrastrukturen.

Zur technischen Steuerung vieler dieser Systeme werden seit langem Informationsund Kommunikationstechnologien eingesetzt, zur Steuerung der Anlagen, zur Überwachung des technischen Betriebs und zum automatisierten oder manuellen Eingriff bei Ausnahmesituationen. Neben dieser technischen Steuerung gibt es auch eine ganze Reihe von Informationssystemen, mit deren Hilfe man die betriebswirtschaftlichen Prozesse abbildet. Dies geschieht für die Bilanzierung und Wartung bestehender Anlagen und Infrastrukturen ebenso wie für die Abrechnungsvorgänge der Dienstleistungen, die von den Versorgungsunternehmen erbracht werden. Mit dem Wandel des Energiesystems muss nun auch durchdacht werden, wie die IKT in Zukunft gestaltet werden kann, um den geänderten Anforderungen gerecht zu werden.

Für die offene Systemintegration der Energielandschaft von morgen muss also eine angemessene IKT Infrastruktur geschaffen werden. Diese wurde auch als „Internet der Energie“ bezeichnet. Die Metapher besagt hierbei im Wesentlichen: So wie das Internet alle Computer über einen Satz offener Standards und Protokolle miteinander kommunizieren lässt, so sollten alle Komponenten des zukünftigen Energiesystems (Erzeuger, Netze, Speicher und Verbraucher) auf der Grundlage standardisierter offener Architekturen untereinander verbunden werden können. Andere verwandte Schlagworte sind „Smart Grid“ oder das „Intelligente Netz“.

Tatsächlich enthält die erwähnte Novelle des Energiewirtschaftsgesetzes vom Sommer 2011 eine ganze Reihe von Aspekten, die direkt mit diesem Thema zu tun haben. 
Das EnWG schafft u. a. einen Rahmen für die Integration von zu- und abschaltbaren Lasten ( $\$ 13$ Abs. 4a EnWG-E), äußert sich über ein sicheres und effizienzorientiertes Smart Metering ( $\$ \$ 21 \mathrm{~b}-\mathrm{i}$ EnWG-E) und über variable Tarife und unterbrechbare Verbrauchseinrichtungen $(\$ 40 \mathrm{~V}, \S 14$ a EnWG-E) und beschreibt schließlich ein Energieinformationssystem unter Netzbetreibern ( $\$ 12$ Abs. 4 EnWG-E). Auch wenn viele dieser Themen erst in noch auszuarbeitenden Verordnungen final geregelt und operationalisiert werden, steht somit doch der große Rahmen fest. Die Transformation ist damit nicht mehr der Ausblick diverser Vordenker, sondern wird in einem konkreten und verbindlichen Gesetz greifbar.

Vor diesem Hintergrund erhält das vorliegende Heft eine unmittelbare Aktualität. Die Beiträge zeigen auf, wie die Transformation wissenschaftlich begleitet und fachlich unterstützt werden kann. In dem Artikel „Methoden zum Management von Informationsquellen für die Unterstützung von Softwareproduktmanagern in der Energiewirtschaft" stellen die Autoren González Vázquez, Sauer und Appelrath einen Referenzmodellkatalog für die Energiewirtschaft vor, der insbesondere Produktmanagern eine Systemunterstützung bei der Fusion der zahlreichen Informationsquellen bieten kann. In dem Modell werden branchenspezifische Anforderungen von Kunden, dem Regulator und den zahlreichen technischen Standards zusammengeführt.

Der Beitrag „Integrierte Informationsversorgung zur Entscheidungsunterstützung in Netzgesellschaften" von Felden und Buder konzipiert ein Referenzmodell zur Ableitung praktischer Handlungsempfehlung für das strategische Anlagenmanagement und validiert dies über Expertengespräche. Es dient gleichzeitig der notwendigen Integration technischer und betriebswirtschaftlicher Artefakte aus der derzeitigen verteilten Datenhaltung als auch als Entscheidungsunterstützung im Kontext der Business Intelligence.

Der Artikel von Flath, Nicolay, Conte, van Dinther und Filipova-Neumann „Clusteranalyse von Smart-Meter-Daten - Eine praxisorientierte Umsetzung“ zeigt auf, wie mithilfe von Daten, die im Smart Metering erhoben werden, neue Dienste und verbesserte Prozesse und Angebote entwickelt werden können. Dabei spielt eine deutlich feinere Kundensegmentierung auf Basis des zeitlichen Verbrauchsverhaltens eine entscheidende Rolle, die durch eine Clusteranalyse in der Business-Intelligence-Umgebung erreicht werden kann. Die Analyse beruht auf realen Daten, die in einem Projekt mit einem lokalen Versorger erhoben wurden.

Im Interview führt Prof. Dr. Henning Kagermann, Präsident der acatech - Deutsche Akademie der Technikwissenschaften, seine Gedanken zum Thema „Smart Grids - Informations- und Kommunikationstechnologie als Schlüsselfaktor für ein zukunftsfähiges Energiesystem“ aus. Und kündigt dabei an, dass acatech an einem Vorschlag für eine deutschlandweite Smart Grid-Plattform nach Muster der Nationalen Plattform Elektromobilität arbeitet, für die Anfang Februar Empfehlungen in Berlin präsentiert werden. Nur so können sich nach Meinung Kagermanns die Akteure auf eine vorausschauende und mit der Politik abgestimmte Industrie- und Forschungspolitik verständigen.

Wir hoffen, dass mit diesem Heft ein Beitrag geleistet wird, der fundierte Forschung mit praxisrelevanten Themen zusammenbringt und so zur Weiterentwicklung dieses so zentralen Themas unserer Energiewirtschaft, aber auch gesamten Volkswirtschaft beitragen kann. Wir wünschen eine anregende Lektüre! 